\title{
Direct transmission vs relay transmission for information dissemination in a MANet: an analytical study
}

\author{
Serge Chaumette \\ LaBRI, University of Bordeaux - France \\ serge.chaumette@labri.fr
}

\author{
Jonathan Ouoba \\ VTT Technical Research Centre of Finland \\ ext-jonathan.ouoba@vtt.fi
}

\begin{abstract}
It is acknowledged that in a mobile environment the wireless technologies that are available at each node can be exploited so as to achieve efficient peer-to-peer communications. Therefore we have developed a multilevel platform the goal of which is to allow a set of mobile terminals to securely communicate in a peer-to-peer manner by using the most appropriate available technology according to the context at hand. The scenario that we have chosen to focus on targets information sharing for collaboration purpose between the mobile nodes of the network. The study of this scenario led us to identify the main operations that are required to achieve it, the central process being the publication of profiles. This operation is meant to allow a node to publish a description (that we call a profile) of the information it is willing to share with the other nodes. Two approaches, direct transmission and relay transmission, are considered so that the publication of profiles can be performed in the most efficient way (according to the available communication technologies and the dynamics of the network). In this paper, we first present the target environments that we consider and the approach that we have chosen to implement in our multilevel platform. We then focus on the publication of profiles and we highlight the two transmission modes (direct transmission and relay transmission) that we have chosen to consider. We analytically study and compare them in terms of the probability to successfully deliver a given message in the target context defined above. We conclude with future research directions.
\end{abstract}

Keywords-MANETs; peer-to-peer; communications; mobility; relay transmission

\section{INTRODUCTION}

The combined and effective use of the different wireless technologies supported by today mobile devices requires detailed analysis as it increases their communication capabilities. Our focus is on the identification of the various opportunities offered by this multi-technology environment in terms of mobile services and applications. Considering these elements, we have proposed a multilevel platform allowing a set of mobile terminals (mobile phones in particular) to securely communicate in peer-to-peer mode, using the most appropriate technology according to the context [1]. The term multilevel refers to the fact that each available (wireless) technology represents a possible level of communication with its own characteristics (range, transmission rate, etc). More concretely,

This work was partly carried out during the tenure of an ERCIM "Alain Bensoussan" Fellowship Programme. The research leading to these results has received funding from the European Union Seventh Framework Programme (FP7/2007-2013) under grant agreement $\mathrm{n}^{\mathrm{o}} 246016$. the scenario is the sharing of data between the entities so that each node of the network can provide information to the others and also look for information it is interested in. We have identified the following main operations to achieve this scenario: the publication of profiles which allows a node to publish the details concerning the type of information it is willing to share; the specification of a set of targets which makes it possible for a node to identify the entities which may meet its needs; the choice of technology which allows a node to select the most appropriate technology to communicate; the security of communications which is intended to prevent the (unauthorized) disclosure of private data. In this paper, we focus on the publication of profiles which is central. To publish the details concerning the type of information (the profile) it is willing to share, a node makes use of the available technologies in a peer-to-peer mode. The potential recipients are thus the nodes which are directly accessible via the available technologies. We consider the following options, from the perspective of the publishing node, for this operation: the direct transmission in which the considered node directly sends the information to the recipients; the relay transmission in which the considered node sends the information to one of its neighbors so that it can retransmit the message to the recipient. Then, the question that we have chosen to study can be synthesized in the following manner: in this multilevel (multi-technology) context, how to decide between the situations where it is preferable to directly send the information to the target recipient and the situations where the use of a relay is more adequate?

The rest of this paper is organized as follows. First, we present the target environment of our multilevel platform and we position our approach. We then describe in more details the publication of profiles to highlight the related key issues. Thereafter, we provide the results of the analytical study that we have conducted regarding the probability of reaching the recipients for the two transmission options. Finally, we present the future research directions that we plan to address based on the first results before concluding.

\section{Context}

\section{A. Target environment}

The Mobile Ah hoc Networks (MANets) are the target environment of the platform in which the publication of 
profiles is performed. Conventionally, a MANet (Mobile Ad hoc Network) is composed of a set of communicating devices which are able to spontaneously interconnect without any preexisting infrastructure; it configures itself on the fly. One of the key issues in this kind of environment is the dissemination of content [2]. Usually, the research related to this key issue focuses on broadcasting with reactive and proactive protocols [3] [4] and on probability-based, neighbor knowledge-based and area-based routing protocols [5] [6]. It is important to note that factors such as the density or the mobility of the nodes in the network as well as the selected execution scenario have an impact on the performance of the routing protocols [7] [8] and the broadcasting methods. Considering the previous elements, we thus believe that it is necessary to decrease, compared to more conventional (infrastructure-oriented) networks, the level of guarantees we expect from MANets [9]. For example, concerning the publication of profiles in our platform, we cannot guarantee that a profile will reach all the nodes of the network. Rather, the goal will be to allow a node to publish its profile to the largest possible set of entities of the network (what depends on its neighborhood). In addition, we have chosen to favor the opportunistic approach that we describe in the next section.

\section{B. The opportunistic approach}

The principle of this approach is to use the mobility of the nodes to deliver the messages. This is known as the storeand-forward technique and the nodes whose mobility is used to forward the messages are called data mules. Due to the unpredictable mobility of the nodes, the delays of transmission are usually long (as the process requires opportunistic interactions) and it is virtually impossible to guarantee that a message will be delivered at a given destination [10].

Although certain guarantees cannot be provided, we believe that these methods, particularly the content-oriented method [11] in which a node sends the relevant requests to the other nodes it encounters to obtain the desired information, can be considered realistic in the context of MANets. By leveraging this approach in our platform, we choose to use the mobility of the entities of the network to disseminate a published profile.

\section{The PUBLICATION OF PROFILES}

We provide the following definitions that we use below for modeling purpose:

- profile $_{n i}$ is the profile that $n_{i}$ is willing to publish;

- techno $_{n_{i}}=T_{n_{i}}$ is the set of wireless technologies that $n_{i}$ can use

- $V_{n_{i}}(t)$ is the set of neighbors of $n_{i}$ at time $t$;

- $P_{n_{i}}$ contains the identifiers of the nodes corresponding to the profiles that $n_{i}$ has received

- $T_{n_{i}, n_{k}}$ is the set of wireless technologies by which a node $n_{k}$ is the neighbor of a node $n_{i}$;

- $\max T_{n_{i}, n_{k}}$ is the technology with the largest coverage area in the set $T_{n_{i}, n_{k}}$;

- $V T_{n_{i}}(t)$ is the set of pairs $\left(n_{k}, T_{n_{i}, n_{k}}\right)$ with $n_{k} \in V_{n_{i}}(t)$ and $T_{n_{i}, n_{k}} \subset T_{n_{i}}$
- $\geq^{c}\left(t_{a}, t_{b}\right)$ represents the fact that the coverage area of the technology $t_{a}$ is at least $c$ times larger than the coverage area of the technology $t_{b}$.

Prior to the initiation of the publication of its profile, we assume that $n_{i}$ has collected information about its current neighbors. At time $t, n_{i}$ publishes its profile. $n_{p}$ and $n_{l}$ are potential recipients of the profile : $n_{p} \in V_{n_{i}}(t), n_{l} \in V_{n_{i}}(t)$, the identifier of $n_{i} \notin P_{n_{p}}$ and the identifier of $n_{i} \notin P_{n_{l}}$. The following steps, represented figure 1 , are considered:

- case 1: transmission by relay. If $n_{i}$ and $n_{l}$ have a common neighbor $\left(V_{n_{i}}(t) \cap V_{n_{l}}(t) \neq \varnothing\right)$, then for each node $n_{m} \in$ $V_{n_{i}}(t) \cap V_{n_{l}}(t)$ we have pairs $\left(n_{m}, T_{n_{i}, n_{m}}\right)$ and $\left(n_{m}, T_{n_{l}, n_{m}}\right)$ which respectively belong to the sets $V T_{n_{i}}(t)$ and $V T_{n_{l}}(t)$. In other words, $n_{m}$ is accessible by $n_{i}$ and $n_{l}$ respectively via the technologies of $T_{n_{i}, n_{m}}$ and $T_{n_{l}, n_{m}}$.

If $\geq^{f}\left(\max _{n_{l}, n_{m}}, \max T_{n_{i}, n_{m}}\right)$ (the value of the factor $f$ will be discussed later) then $n_{i}$ sends its profile to $n_{l}$ which retransmits it to $n_{m}$ via $\max _{n_{l}, n_{m}}$ (we assume that, in mobility situations, the greater the coverage area of a technology, the more chance to reach a recipient by using it).

- case 2: direct transmission. If the node $n_{p}$ is an isolated node (it only has $n_{i}$ as a neighbor), then $n_{i}$ sends its profile to $n_{p}$ via $\operatorname{maxT}_{n_{i}, n_{p}}$.

- case 3: default case. If a node $n_{l}$ (neighbor of $n_{i}$ ) was not considered (with the 2 previous steps), then $n_{i}$ sends its profile to $n_{l}$ via $\max _{n_{i}, n_{l}}$.

In order to demonstrate that the steps outlined above enable a node to publish its profile (in peer-to-peer mode) among the largest possible set of nodes, it is necessary to determine the value of the factor $f$ introduced above.

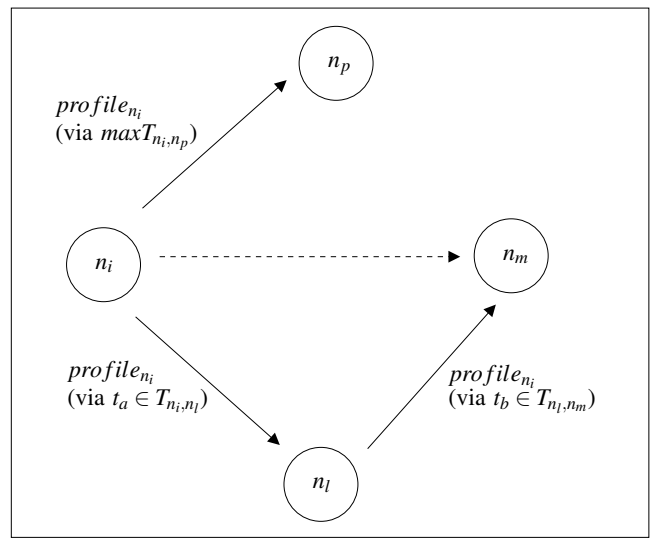

Fig. 1. Direct transmission and relay transmission of a profile.

\section{ANALYTICAL STUDY}

During the publication of its profile by a given node $n_{i}$, a choice must be made, for each potential recipient, between the direct mode and the relay mode for the transmission (in order to reach the largest possible set of entities of the network). It is then a question of determining the value of the factor $f$ as mentioned in the previous section which allows to make a relevant choice between the two transmission options. To this 
end, we first evaluate the probability $\left(p_{1}\right)$ for a node to leave the coverage area of a given technology. Then, we evaluate the success probability $\left(p_{2}\right)$ of a message transfer between 2 nodes via another node (relay transmission). Finally, we compare the results to draw a first conclusion. Prior to these calculations, we present the general assumptions that we chose to retain.

\section{A. Assumptions}

For the analytical study, we have chosen to make the following assumptions:

- the coverage areas of the available (wireless) technologies are circular with an omni-directional propagation scheme. This approach is the most common in the community.

- the required delay so that a sent message reaches its recipient is no more than one unit of time.

- the initiator node does not move between the time it has finished collecting the information about its neighbors and the time it starts the transmission (for the publication of profiles).

\section{B. Calculations of probabilities and comparison}

1) Probability $p_{1}$ for a node to leave a coverage area: As presented figure 2, there are two nodes, namely 1 and 2, in a given area. We assume that the node 1 is equipped with a wireless technology $t_{1}$ whose coverage area is represented by the circle with the radius $r$ (area $S_{1}$ ). We also assume that the node 2 moves at a speed $v$ which allows it to cover a distance $d_{\delta}$ per unit of time (with $d_{\delta}<r$ ). The small circle (represented with dashes) is a circle whose center is the point where the node 2 is located and whose radius is $d_{\delta}$. This circle corresponds to the area reachable by the node 2 in one unit of time. We define, within the coverage area of the technology $t_{1}$, a risk area which is the space between the perimeter of the circle defining the coverage area of $t_{1}$ and the perimeter of the dotted circle (whose area is denoted by $S_{1}^{\prime}$ ). The difference in radius between the two circles is equal to $d_{\delta}$. This risk zone is an area from which the node 2 can (potentially) move during one unit of time and be out of reach of the technology $t_{1}$ (thus moving a distance $d_{\delta}$ ).

Let $Q$ be the event "the node 2 is accessible by the node 1 and it is within the risk zone of the technology $t_{1}$ " and $R$ be the event "the node 2 leaves the coverage area of $t_{1}$ when it is in the risk zone". The probability that the node 2 leaves the coverage area of the technology $t_{1}$ (equipping the node 1) corresponds to $p(Q \cap R)$. It is therefore necessary to calculate the probability $p(Q)$ of occurrence of the event $Q$ and the probability $p(R)$ of occurrence of the event $R$. Obviously, it is possible to establish the following relations:

$$
\begin{aligned}
& \text { - } p(Q)=1-\frac{S_{1}^{\prime}}{S_{1}} \text { with } S_{1}=\pi r^{2} \text { and } S_{1}^{\prime}=\pi\left(r-d_{\delta}\right)^{2} \\
& \Rightarrow p(Q)=1-\frac{\pi\left(r-d_{\delta}\right)^{2}}{\pi r^{2}}=\frac{2 r d_{\delta}-d_{\delta}^{2}}{r^{2}}
\end{aligned}
$$

We then define an orthonormal system (figure 2) centered at $A$ (the point where the node 1 is located), the abscissa axis of which is represented by the line $(A B)$. $B$ is the point where the node 2 is located (in the risk zone). The abscissa axis is oriented in the direction $A$ to $B$. The coordinates of
$A$ and $B$ are: $A(0,0)$ and $B(x, 0)$ with $x \in\left[r-d_{\delta}, r\right]$. In order to determine $p(R)$, it is necessary to evaluate the average value $\left(\theta_{m}\right)$ of the angle $\theta$ according to the possible values for the abscissa of $B\left(x \in\left[r-d_{\delta}, r\right]\right)$. Indeed, $\theta$ is the angular portion which, if node 2 moves in it, will cause it to leave the coverage area of node 1.

First, we can determine the coordinates of the points $M$ and $N$ which are located at the intersection of the circle representing the coverage area of the technology $t_{1}$ and the circle with the radius $d_{\delta}$. Let $\left(x_{m}, y_{m}\right)$ and $\left(x_{n}, y_{n}\right)$ be the coordinates of $M$ and $N$. It is possible to establish the following relations:

$$
\begin{aligned}
& \text { - }\left\{\begin{array} { l } 
{ \| \vec { A M } \| = r } \\
{ \| \vec { B M } \| = d _ { \delta } }
\end{array} \Rightarrow \left\{\begin{array}{l}
\sqrt{x_{m}^{2}+y_{m}^{2}}=r \\
\sqrt{\left(x_{m}-x\right)^{2}+y_{m}^{2}}=d_{\delta}
\end{array}\right.\right. \\
& \text { - we obtain } d_{\delta}^{2}-\left(x_{m}-x\right)^{2}=r^{2}-x_{m}^{2} \\
& \Rightarrow d_{\delta}^{2}+2 x_{m} x-x^{2}=r^{2} \\
& \Rightarrow x_{m}=\frac{x^{2}+r^{2}-d_{\delta}^{2}}{2 x}
\end{aligned}
$$

- as we know that $y_{m}^{2}=r^{2}-x_{m}^{2}$, we have

$$
y_{m}=\frac{1}{2 x} \sqrt{\left(2 r x+r^{2}+x^{2}-d_{\delta}^{2}\right)\left(2 r x-r^{2}-x^{2}+d_{\delta}^{2}\right)}
$$

Similarly, it is possible to determine the following relations for the point $N$ :

$$
\text { - }\left\{\begin{array}{l}
x_{n}=\frac{x^{2}+r^{2}-d_{\delta}^{2}}{2 x} \\
y_{n}=-\frac{1}{2 x} \sqrt{\left(2 r x+r^{2}+x^{2}-d_{\delta}^{2}\right)\left(2 r x-r^{2}-x^{2}+d_{\delta}^{2}\right)}
\end{array}\right.
$$

With the relation between $\|\overrightarrow{B M}\|$ and $\|\overrightarrow{B N}\|$ and $\cos (\theta)$ :

- we know that $\langle\overrightarrow{B M} \mid \overrightarrow{B N}\rangle=\| \overrightarrow{B M}|| \cdot|| \overrightarrow{B N}|| \cdot \cos (\theta)$ as $\overrightarrow{B M}\left(x_{m}-x, y_{m}\right)$ and $\overrightarrow{B N}\left(x_{n}-x, y_{n}\right)$ so we can establish $\left(x_{m}-x\right)\left(x_{n}-x\right)+y_{m} y_{n}=d_{\delta}^{2} \cos (\theta)$ $\Rightarrow \frac{x^{4}-2 r^{2} x^{2}+\left(r^{2}-d_{\delta}^{2}\right)^{2}}{2 x^{2}}=d_{\delta}^{2} \cos (\theta)$ if we set $X^{2}=x^{2}, a=2 r^{2}, b=\left(r^{2}-d_{\delta}^{2}\right)^{2}$ et $c=2 d_{\delta}^{2}$ then we have $\cos (\theta)=\frac{X^{2}-a X+b}{c X}$

- $\theta=\arccos \left(\frac{X^{2}-a X+b}{c X}\right)$ with $X \in\left[\left(r-d_{\delta}\right)^{2}, r^{2}\right]$ therefore the average value of $\theta$ by considering the range $\left[\left(r-d_{\delta}\right)^{2}, r^{2}\right]$ is

$\theta_{m}=\frac{1}{2 r d_{\delta}-d_{\delta}^{2}} \int_{\left(r-d_{\delta}\right)^{2}}^{r^{2}} \arccos \left(\frac{X^{2}-a X+b}{c X}\right) \mathrm{d} X$

With the Riemann sum we can obtain an approximate value of $\theta_{m}$ :

$$
\begin{aligned}
& -\theta_{m}=\frac{1}{n} \sum_{k=1}^{n} f\left(\left(r-d_{\delta}\right)^{2}+k \frac{2 r d_{\delta}-d_{\delta}^{2}}{n}\right) \quad \text { with } \\
& f(x)=\arccos \left(\frac{x^{2}-a x+b}{c x}\right), x \in\left[\left(r-d_{\delta}\right)^{2}, r^{2}\right] \text { and } n \text { a } \\
& \text { selected natural number (the higher the value of } \mathrm{n} \text {, the } \\
& \text { closer to the real value the calculation) }
\end{aligned}
$$

Due to the independent nature of the events $Q$ and $R, p(Q \cap$ $R)=p(Q) \times p(R)$. According to the previous calculations, we have $p(R)=\frac{\theta_{m}}{2 \pi}$ (i.e. probability to leave the coverage area from the risk zone). We also know that $p(Q)=\frac{2 r d_{\delta}-d_{\delta}^{2}}{r^{2}}$. We 
can then conclude that $p_{1}=p(Q \cap R)=\frac{\theta_{m}\left(2 r d_{\delta}-d_{\delta}^{2}\right)}{2 \pi r^{2}}$

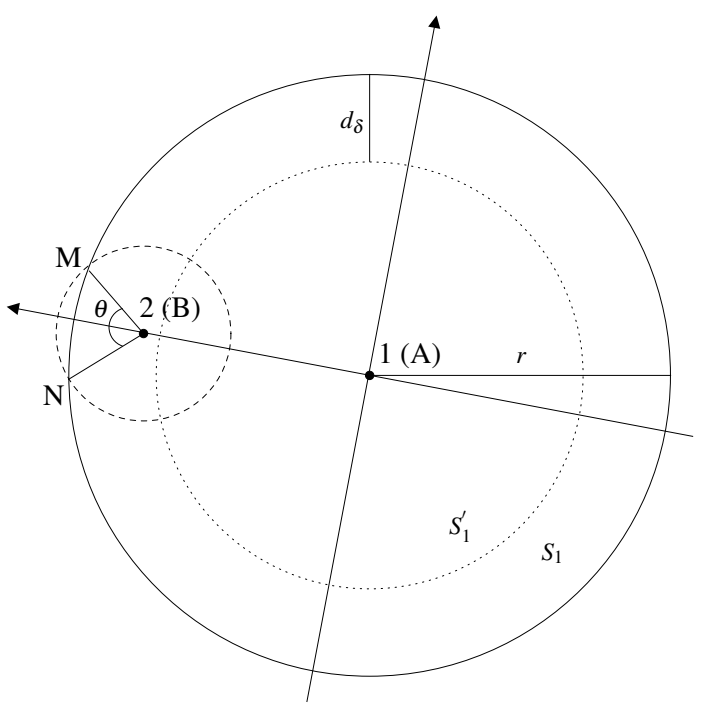

Fig. 2. Possibilities for a node to leave a coverage area.

2) Probability $p_{2}$ of success for a message transfer between three nodes: Let consider three nodes, 1, 2 and 3 , in a given area (figure 3 ). We assume that the node 1 is equipped with the wireless technologies $t_{1}$ and $t_{2}$ whose coverage areas are respectively represented by the circle $\mathscr{C}_{1}$ with radius $r_{1}$ (this area is denoted $S_{1}$ ) and the circle $\mathscr{C}_{2}$ with radius $r_{2}$ (this area is denoted $S_{2}$ ). The node 3 is equipped with the technology $t_{3}$ whose coverage area is represented by the circle $\mathscr{C}_{3}$ with radius $r_{3}$ (this area is denoted $S_{3}$ ). We also assume that $r_{1}<r_{2}<r_{3}$ (so that the relay transmission procedure described in section III makes sense) and that the nodes 2 and 3 can move at a speed $v$ which allows them to cover a distance $d_{\delta}$ per unit of time. We define, within the coverage area of each technology, a risk zone which is the space between the perimeter of the circle representing the coverage area and the perimeter of the dotted circle. This risk zone is an area from which a node can leave the coverage area of the considered technology if it moves during one unit of time (according to the distance $d_{\delta}$ defined above). The areas represented by the dotted circles are respectively $S_{1}^{\prime}, S_{2}^{\prime}$ and $S_{3}^{\prime}$ for the circles $\mathscr{C}_{1}, \mathscr{C}_{2}$ and $\mathscr{C}_{3}$.

At time $t$, the node 2 is located in the zone $\mathscr{C}_{1}$ and the node 3 is located in the zone $\mathscr{C}_{2}$. The operation to perform is as follows: the node 1 must transmit a message $m$ (in one unit of time so that we can take into account in the calculations the potential movement of the nodes 2 and 3 at the speed $v$ ) to the node 2 via the node 3 . It is a question of determining the probability that the three nodes are in a configuration in which the message $m$ is successfully transmitted (despite the possible movements of the nodes).

Let $Q$ be the event "the node 3 stays in the zone $\mathscr{C}_{2}$ after one unit of time" and $R$ be the event "the node 2 stays in the zone $\mathscr{C}_{3}$ after one unit of time". The probability that we want to determine is the probability $p(Q \cap R)$. It is therefore necessary to calculate the probability $p(Q)$ of occurrence of the event $Q$ and the probability $p(R)$ of occurrence of the event $R$. Considering the areas $S_{2}, S_{3}, S_{2}^{\prime}$ and $S_{3}^{\prime}$, it is possible to establish the following relations:

$$
\begin{aligned}
\text { - } & p(Q)=\frac{S_{2}^{\prime}}{S_{2}} \text { with } S_{2}=\pi r_{2}^{2} \text { et } S_{2}^{\prime}=\pi\left(r_{2}-d_{\delta}\right)^{2} \\
& \Rightarrow p(Q)=\frac{\pi\left(r_{2}-d_{\delta}\right)^{2}}{\pi r_{2}^{2}}=\frac{r_{2}^{2}-2 r_{2} d_{\delta}+d_{\delta}^{2}}{r_{2}^{2}} \\
\text { - } & p(R)=\frac{S_{3}^{\prime}}{S_{3}} \text { with } S_{3}=\pi r_{3}^{2} \text { et } S_{3}^{\prime}=\pi\left(r_{3}-d_{\delta}\right)^{2} \\
\Rightarrow & p(R)=\frac{\pi\left(r_{3}-d_{\delta}\right)^{2}}{\pi r_{3}^{2}}=\frac{r_{3}^{2}-2 r_{3} d_{\delta}+d_{\delta}^{2}}{r_{3}^{2}}
\end{aligned}
$$

- the events $Q$ and $R$ are independent

$$
\begin{aligned}
& \text { then } p_{2}=p(Q \cap R)=p(Q) \times p(R)= \\
& \left(\frac{r_{2}^{2}-2 r_{2} d_{\delta}+d_{\delta}^{2}}{r_{2}^{2}}\right) \cdot\left(\frac{r_{3}^{2}-2 r_{3} d_{\delta}+d_{\delta}^{2}}{r_{3}^{2}}\right)
\end{aligned}
$$

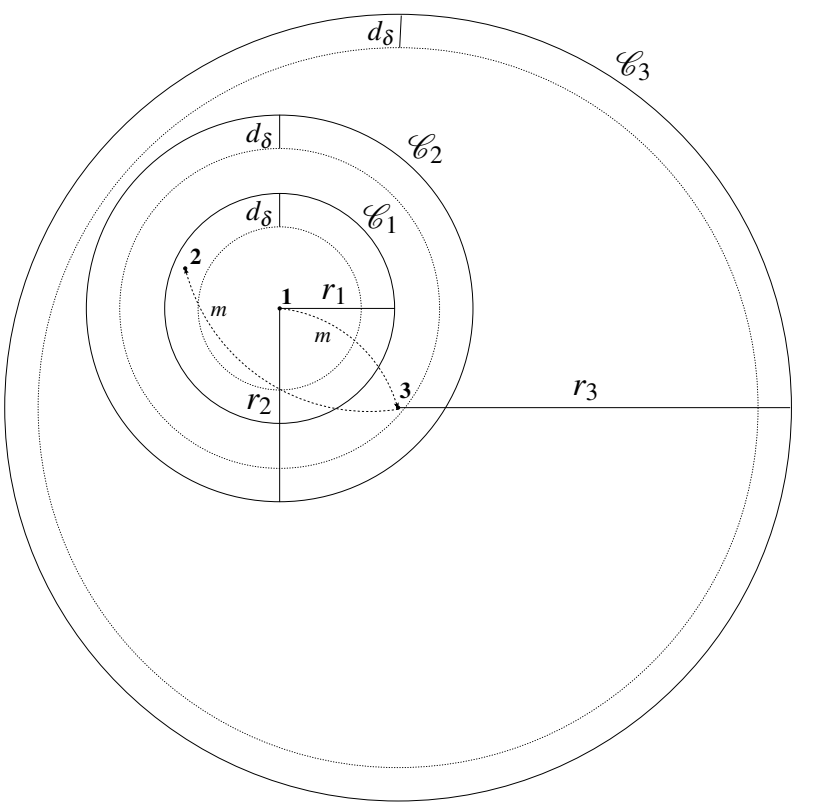

Fig. 3. Possibilities of transfer of a message between three nodes.

3) Comparison between the two procedures of transmission: In the case a node $n_{i}$ wishes to transmit a message $m$ to an accessible node $n_{j}$ in a mobile context, there are two possibilities for $n_{i}$. The sending node $\left(n_{i}\right)$ can either directly send the message to the target (via a technology $t_{1}$ ) or use a relay node $n_{k}$ (equipped with a technology $t_{3}$ whose coverage area is larger) to retransmit the message to the node $n_{j}$. In this relay mode, the node $n_{i}$ sends the message to the node $n_{k}$ via a technology $t_{2}$. It is assumed that the node $n_{i}$ is equipped with the technologies $t_{1}$ and $t_{2}$, the node $n_{j}$ is equipped with the technologies $t_{1}$ and $t_{3}$, while the node $n_{k}$ is equipped with the technologies $t_{2}$ and $t_{3}$.

Considering the probabilities ( $p_{1}$ and $p_{2}$ ) that we have calculated above, it is possible to determine the most appropriate method to transmit a message in the considered context. Indeed, if the probability for the node $n_{j}$ to leave the coverage area of the technology $t_{1}$ is greater than the probability for the node $n_{k}$ to be in an area that allows it to retransmit the message, the relay method should be privileged. 
Thus, when a node $n_{i}$ must determine the most appropriate mode to send a message to a node $n_{j}$, after considering the coverage areas of the technologies $t_{1}, t_{2}$ and $t_{3}$, it evaluates the probabilities $p_{1}$ and $p_{2}$ (using the available information concerning the technologies). Based on the results, the choice of the transmission mode can then be made.

In order to provide a first idea of the evolution of the probabilities for both transmission modes, we have drawn the graphic presented figure 4 . It represents the difference between the relay mode and direct mode in terms of probability of success in the transmission of a message with a specific scenario. $r_{1}, r_{2}$ and $r_{3}$ respectively represent the radius of the coverage areas for the technologies $t_{1}, t_{2}$ and $t_{3}$. In addition, we have made the following assumptions for this evaluation (the distances are in meters and the unit of time is the second):

- the value of $r_{1}$ is fixed to 15 as it corresponds to the coverage area of Bluetooth in the equipments that we used in the development of our multilevel platform [1];

- $r_{2}$ is defined as a multiple of $r_{1}$ according to a parameter $\alpha$ which varies between 1 and 5;

- $r_{3}$ is defined as a multiple of $r_{2}$ according to a parameter $\beta$ which varies between 1 and 5;

- the value of $d_{\delta}$, which is the distance that a node can cover in one unit of time, is fixed to 1.4 as it corresponds to the average speed $(1.4 \mathrm{~m} / \mathrm{s})$ at which humans walk in the absence of external constraints [12].

In the studied case (relevant in our context), we can conclude that 3 (the use of the relay mode becomes relevant when $\alpha$ is at least equal to 1.5 and $\beta$ at least equal to 2 ) is the value of the factor $f$ which can be used to choose between the two possibilities (direct transmission or relay transmission).

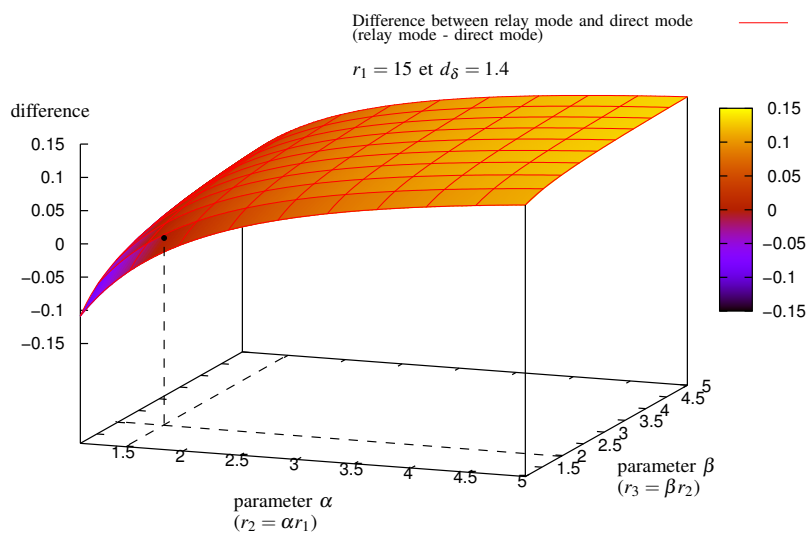

Fig. 4. Difference for the probability of success between the two methods

\section{CONCLUSION}

We have presented the study of the publication of profiles in the context of the framework that we have proposed. The publication of profiles is meant to allow a mobile node to efficiently publish (i.e as much as possible) in its neighborhood the information it is willing to provide to the other entities of the network.

Our work lead us to identify two main options regarding the transmission mode for the publication of profiles: the direct transmission and the the relay transmission. In the direct mode, the sending node directly transmits the message to the recipient while in the relay mode, it makes us of a relay node to reach the recipient. According to the characteristics of the wireless technologies supported by the nodes, the analytical study that we have conducted allowed us to determine the formulas of the probabilities $p_{1}$ and $p_{2}$ that respectively represent the probabilities of success for a transmission in direct mode and for a transmission in relay mode. In addition, through the analysis of a specific case, we have provided a first example of the comparative evolution for the two probabilities (so that a relevant choice can be made between the two options). In our future work we will refine the proposed model so as to take into account specific mobility models and to integrate parameters such as energy-efficiency. We also plan to conduct experiments for a practical evaluation of our approach.

\section{REFERENCES}

[1] S. Chaumette and J. Ouoba, "Multilevel and Secure Services in a Fleet of Mobile Phones: The Multilevel Secured Messaging Application (MuSMA)," in Proceedings of the Fourth International Conference on Mobile Computing, Applications, and Services, ser. MobiCASE 2012. Springer, Oct. 2012, pp. 169-185.

[2] H. Li, K. Bok, K. Chung, and J. Yoo, "An efficient data dissemination method over wireless ad-hoc networks," Wireless Personal Communications, pp. 1-20, 2014.

[3] T. Clausen and P. Jacquet, "Optimized link state routing protocol (OLSR)," RFC 2501, Internet Engineering Task Force, 2003.

[4] Z. J. Haas, M. R. Pearlman, and P. Samar, "The Zone Routing Protocol (ZRP) for Ad Hoc Networks," IETF MANET Working Group, Tech. Rep., 2002.

[5] A. Al Hanbali, M. Ibrahim, V. Simon, E. Varga, and I. Carreras, "A survey of message diffusion protocols in mobile ad hoc networks," in Proceedings of the 3rd International Conference on Performance Evaluation Methodologies and Tools, ser. ValueTools '08. Brussels, Belgium: ICST (Institute for Computer Sciences, Social-Informatics and Telecommunications Engineering), 2008, pp. 82:1-82:16.

[6] P. Nand and S. Sharma, "Analytical study of broadcast in mobile adhoc network," International Journal of Computer Applications, vol. 19, no. 8, pp. 7-12, April 2011, published by Foundation of Computer Science.

[7] N. I. Sarkar and W. G. Lol, "A study of manet routing protocols: Joint node density, packet length and mobility," in Proceedings of the The IEEE symposium on Computers and Communications, ser. ISCC '10. Washington, DC, USA: IEEE Computer Society, 2010, pp. 515-520.

[8] E. Nordstrom, P. Gunningberg, C. Rohner, and O. Wibling, "A Comprehensive Comparison of MANET Routing Protocols in Simulation, Emulation and the Real World," Uppsala University, 2006.

[9] S. Chaumette, "Can highly dynamic mobile ad hoc networks and distributed mems share algorithmic foundations?" in Proceedings of the 2012 Second Workshop on Design, Control and Software Implementation for Distributed MEMS, ser. DMEMS '12. Washington, DC, USA: IEEE Computer Society, 2012, pp. 66-73.

[10] A. Benchi, F. Guidec, and P. Launay, "A Message Service for Opportunistic Computing in Disconnected MANETs," in Proceedings of DAIS'12, Stockholm, Suède, Jun. 2012, pp. 118-131.

[11] Y. Mahéo, N. Le Sommer, P. Launay, F. Guidec, and M. Dragone, "Beyond Opportunistic Networking Protocols: a Disruption-Tolerant Application Suite for Disconnected MANETs," in Proceedings of the 4th Extreme Conference on Communication (ExtremeCom'12). Zurich, Suisse: ACM, Mar. 2012, pp. 1-6.

[12] B. J. Mohler, W. B. Thompson, S. H. Creem-Regehr, H. L. J. Pick, and W. H. Warren, "Visual flow influences gait transition speed and preferred walking speed," Experimental Brain Research, pp. 1432-1106, 2007. 